\title{
An Experimental Study of Quinine Blindness
}

\author{
T. A. S. BUCHANAN, R. W. LYNESS, A. D. COLLINS, T. A. GARDINER and \\ D. B. ARCHER
}

Belfast

\begin{abstract}
Summary
An experimental model of quinine induced blindness is presented. Electrophysiological, angiographical and morphological examinations were made. The occurrence of blindness and any recovery from blindness was dependent upon the dose of quinine taken. As no evidence of acute retinal ischaemia was found it is concluded that quinine is retinotoxic.
\end{abstract}

\begin{abstract}
Despite the many clinical ${ }^{1}$ and experimental ${ }^{2}$ reports of quinine blindness providing much detailed evidence and analysis, the first line of clinical therapy continues to be directed at preventing retinal vasoconstriction either using vasodilator drugs or interfering with the sympathetic nerve supply by blocking a stellate ganglion. ${ }^{3,4}$ Our experiment was designed to create an animal model which would allow us to study the genesis of quinine blindness electrophysiologically, fluorescein angiographically and morphologically. Our intention was that by correlating these findings we might identify the site and mode of action of quinine, so that rational therapeutic regimens could be designed and tested. We present some of our preliminary observations.
\end{abstract}

\section{Material and Methods}

Specified pathogen free cats both sexes, aged approximately one year and weighing between $2-4 \mathrm{~kg}$ were identified by tattoo and maintained on proprietary cat foot and water ad lib ... The cats were anaesthetised using Xylazine, Ketamine and ET1431 and set up for monocular recording (left eye) of the Electroretinogram (ERG) and Visual
Evoked Response (VER) in a darkened room. Electrode placements were: ERG cornea, VER posterior coronal suture, ground mid frontal and reference neck muscle. The recording convention was for positive upwards in both ERG and VER tracings. The ERG stimulus was delivered by a stroboscopic lamp placed $10 \mathrm{~cm}$ in front of the left eye, and the VER stimulus by light emitting diodes placed $1 \mathrm{~cm}$ in front of the left eye. The ERG and VER signals were amplified using standard physiological amplifiers. Testing of the ERG and VER proceeded under the control of an interactive computer programme. Results were saved on floppy diskettes for subsequent plotting and analysis. Additional observations included blood pressure, heart rate and respiratory rate. All observations and recordings were made at half hourly intervals.

Fourteen cats were anaesthetised and ERG and VER recordings were made to determine the normal range of the ERG A and $B$ waves and the morphology of the VER (Fig. 1). The cats were allowed to recover and at a later date were given oral doses of quinine sulphate. After 2 hours they were anaesthetised and observations commenced. During the acute experiments, when quinine was given, flourescein angiography was performed on the right eye.

After the acute experiment the cats were allowed

From Department of Ophthalmology, Eye and Ear Clinic, Royal Victoria Hospital, Belfast BT126BA, Northern Ireland.

Correspondence to: T. A. S. Buchanan, Department of Ophthalmology, Eye and Ear Clinic, Royal Victoria Hospital, Belfast BT12 6BA.

Presented at the Annual Congress of the Ophthalmological Society of the United Kingdom, April 1987. 
ERG

VER
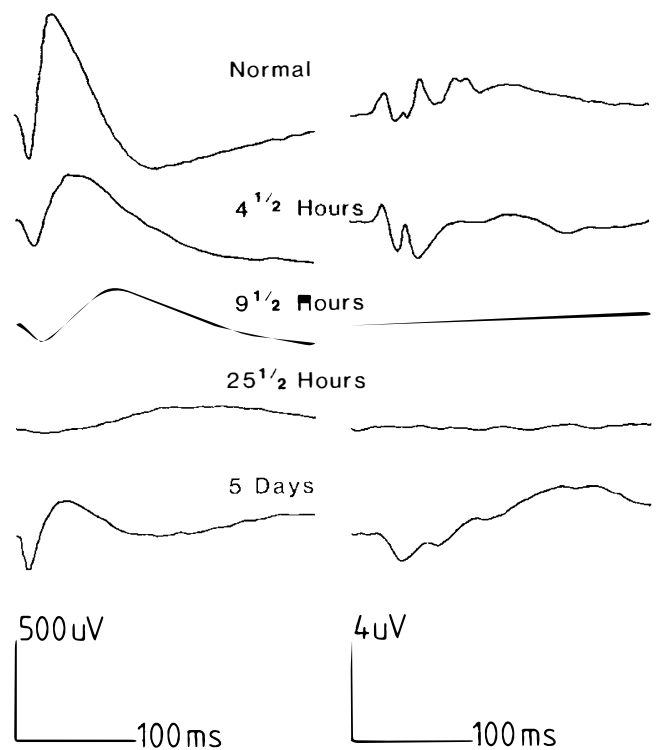

Fig. 1. ERG and VER recordings from a normal cat followed by a sequence of ERG and VER recordings from a cat given $104 \mathrm{mg} / \mathrm{kg}$ of quinine. The elapsed time between ingestion and recording is indicated.

to recover. At intervals after the acute experiment the cats were re-anaesthetised, further ERG and VER recordings made and fluorescein angiography performed.

Eventually all cats were sacrificed and the eyes removed and placed in either buffered formalin or glutaraldehyde. Tissue in formalin was processed to paraffin embedding and standard Haematoxylin and Eosin (H \& E) histological sections made. Tissue in glutaraldehyde was postfixed in 2 per cent osmium tetroxide in $0.1 \mathrm{M}$ cacodylate buffer, dehydrated in ethanol and embedded in TAAB resin. Thin sections were examined electron microscopically.

\section{Results}

The ERG and VER recordings taken from the cats under anaesthesia without quinine showed a consistent pattern. The cats given oral quinine sulphate then anaesthetised 2 hours later showed a reproducible pattern of visual deterioration, during which the other parameters of blood pressure, heart rate and respiratory rate remained within normal limits.

With a given dose of $104 \mathrm{mg} / \mathrm{kg}$ of quinine the ERG and VER recordings (Fig. 1) performed 4-1/2 hours after ingestion revealed a slight increase in ERG $B$ wave latency and a normal VER. The ERG A and $B$ waves became increasingly delayed yet maintained relatively normal amplitudes over the next 8 hour period. By contrast, the VER became delayed and diminished rapidly to the base line within the next 5 hour period. Finally, at 14 hours after ingestion of quinine the ERG had stabilised being grossly delayed and with a subnormal amplitude. The VER was still flat.

The ERG recorded 4 days later had recovered to within the normal range of amplitudes but demonstrated a slightly delayed B wave latency. The VER was present but the first positive peak was still poorly defined and this was interpreted as a delayed response. This electrophysiological pattern of visual deterioration was evident in all cats receiving a dose of quinine greater than 100 $\mathrm{mg} / \mathrm{kg}$. Above $115 \mathrm{mg} / \mathrm{kg}$ of quinine we found no evidence of visual recovery. Cats receiving doses of quinine less than $100 \mathrm{mg} / \mathrm{kg}$ quinine did not go blind.

Fluorescein angiography performed during the acute experiment showed no evidence of significant retinal artery constriction, insufficiency or leakage. Fluorescein angiography performed when reviewing the cats $13-15$ days post-acute experiment showed retinal arteriolar narrowing and at 21 days post-acute experiment one cat subjected to $254 \mathrm{mg} / \mathrm{kg}$ of quinine failed to demonstrate retinal artery perfusion by fluorescein angiography.

Light and electron microscopy of eyes taken both during the acute experiments and over subsequent days all showed retinal extracellular oedema (Fig. 2) with varying degrees of ganglion and neuronal cell death. There was no evidence of any retinal vascular abnormality in eyes taken during or shortly after the acute experiment, however, cats surviving 14 days or more demonstrated marked retinal vascular narrowing. In these eyes there was evidence of retinal architectural collapse and early gliosis.

\section{Discussion}

We have made several deductions from the animal model which we believe have clinical relevance: 


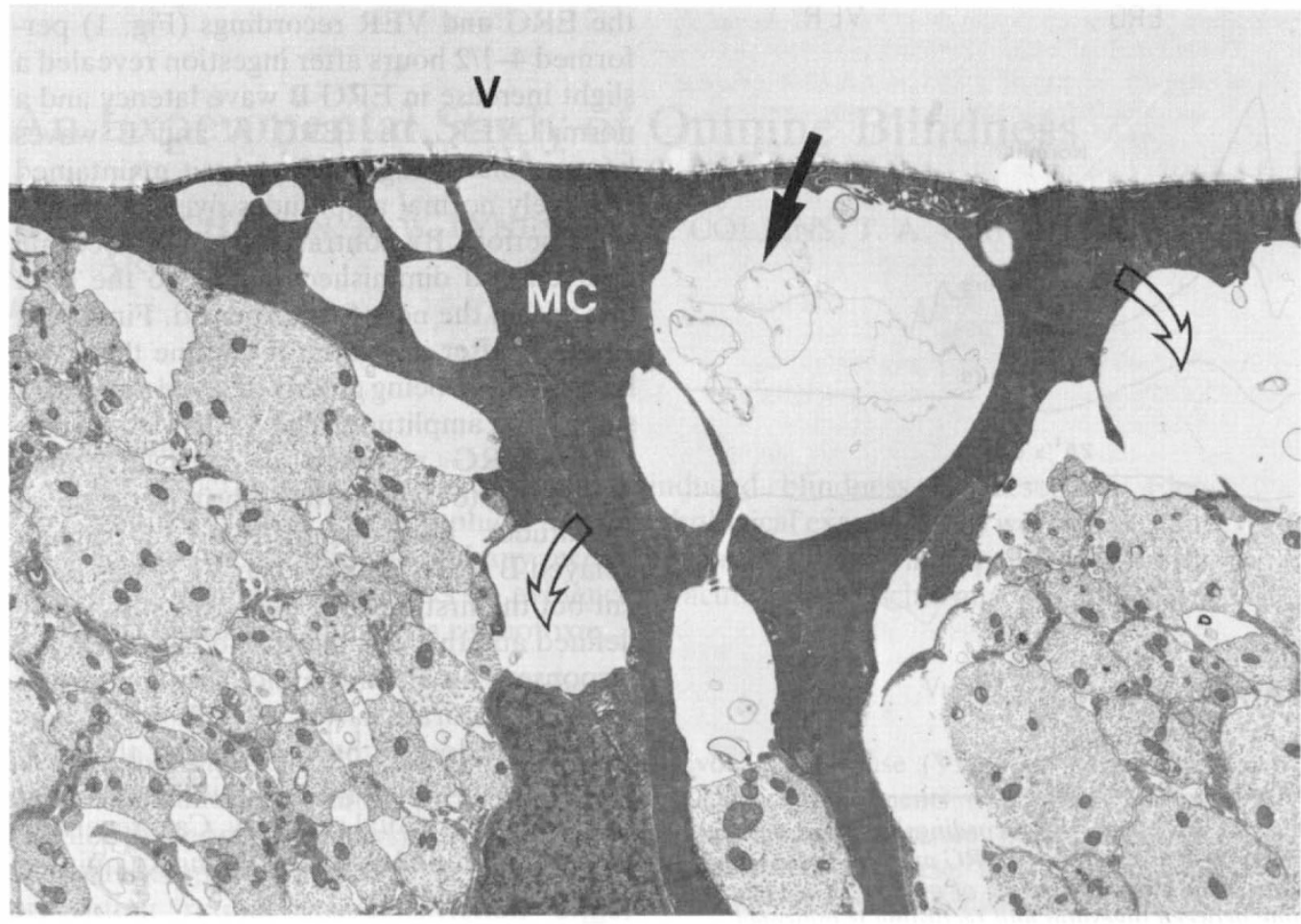

Fig. 2. Electron micrograph of cat retina showing extracellular oedema. The fluid has accumulated in the inner retina (solid arrow) causing enlargement of the extracellular space between the end feet of the Muller cells (MC). Fluid is also present at the interface between the nerve fibre bundles and the surrounding glial processes (hollow arrows). Vitreous body (V). Mag. $\times 4,500$

(1.) We have succeeded in creating an animal model of quinine blindness which mimics the pattern of electrophysiological, angiographical and morphological change seen in patients with a quinine overdose.

(2.) We have been unable to document any evidence that acute ischaemia plays a role in the visual loss experienced by the experimental animal.

(3.) The electrophysiological changes seen after drug administration are most likely due to retinal ganglion, bipolar and photoreceptor cell dysfunction.

(4.) The principal morphological change is an acute accumulation of extracellular fluid with subsequent degeneration of retinal ganglion, bipolar and photoreceptor cells.
(5.) The cat appears to be a suitable model for further investigation of the treatment of acute blindness and further work is planned to evaluate the usefulness of superior cervical ganglion block, corticosteroids and hyperosmolar agents in these animals.

\section{References}

${ }^{1}$ Zahn JR, Brinton GF, Norton E: Ocular quinine toxicity followed by electroretinogram, electrooculogram, and pattern visually evoked potential. Am J Optometry \& Phy Optics 1981, 58: 492-8.

${ }^{2}$ Cibis, GW, Burian HM, Blodi FC: Electroretinogram changes in acute quinine poisoning. Arch Ophthalmol 1973, 90: 307-9.

${ }^{3}$ Thomas D: Forced acid diuresis and stellate ganglion block in the treatment of quinine poisoning. Anaesthesia 1984, 39: 257-60.

${ }^{4}$ Browne GF and Coppel DL: Management of quinine overdose. Human Toxicol 1984, 3: 399-402. 